\title{
Zero-Bending Piezoelectric Micromachined Ultrasonic Transducer (pMUT) With Enhanced Transmitting Performance
}

\author{
Tao Wang, Member, IEEE, and Chengkuo Lee, Member, IEEE
}

\begin{abstract}
A piezoelectric micromachined ultrasonic transducer (pMUT) has enabled numerous exciting ultrasonic applications. However, residual stress and initial buckling may worsen the transmitting sensitivity of a pMUT, and also limit its application and commercialization. In this paper, we report a new innovative pMUT with a perfectly flat membrane, i.e., zero-bending membrane. Leveraging on the stress-free AIN thin film, framelike top electrode layout, and integrated vacuum cavity, the initial deflection of suspended membrane is significantly suppressed to only $0.005 \%$. The transmitting sensitivity of the zero-bending pMUT is measured as $123 \mathrm{~nm} / \mathrm{V}$ at a resonant frequency of $2.21 \mathrm{MHz}$, which is $450 \%$ higher than that of the reference pMUT with slightly non-zero initial deflection. Compared with the simulation results, the measured data of zero-bending pMUT achieve $94.5 \%$ of its ideal transmitting sensitivity. It is solid evidence that our approach is an effective and reliable way to overcome the residual stress and the initial buckling issue.

[2015-0093]
\end{abstract}

Index Terms-Piezoelectric micromachined ultrasonic transducer (pMUT), MEMS, piezoelectric, ultrasound, transmitting sensitivity, bio-medical.

\section{INTRODUCTION}

$\mathbf{U}$ LTRASONIC transducers are widely used for generating and receiving ultrasound waves. Various applications are realized with the ultrasonic transducers, such as noninvasive imaging [1], [2], range finding [3]-[5], and flow velocity sensing [6], [7]. In recent years, advanced MEMS technology has made the ultrasonic transducers to be much smaller, cheaper and consume less power. Novel applications have also emerged based on such micromachined ultrasonic transducers (MUTs). 3-D ultrasonic image can be obtained without mechanical scanning, which has high potential to be the next-generation ultrasonic imaging technology [8]. A miniaturized needle-like MUT is also developed, and it can be put into blood vessels during surgery for intravascular imaging [9]. Besides the imaging related applications,

Manuscript received April 6, 2015; revised July 13, 2015; accepted August 13, 2015. Date of publication September 3, 2015; date of current version November 25, 2015. This work was supported in part by the Science and Engineering Research Council through the Agency for Science, Technology, and Research, Singapore, under Grant 1220103064; and in part by the NRF-CRP001-057 Competitive Research Program entitled Self-Powered Body Sensor Network for Disease Management and Prevention-Oriented Healthcare, through the National Research Foundation-Prime Minister's Office, Singapore, under Grant R-263-000-A27-281.

The authors are with the Department of Electrical and Computer Engineering, National University of Singapore, Singapore 117576 (e-mail: elewt@nus.edu.sg; elelc@nus.edu.sg).

Color versions of one or more of the figures in this paper are available online at http://ieeexplore.ieee.org.

Digital Object Identifier 10.1109/JMEMS.2015.2472958 researchers have also integrated the MUTs into mobile phone to demonstrate airborne gesture recognition [10]. After the touchscreen, airborne gesture recognition may possibly be the next-generation approach of interacting with electronic devices remotely. Beyond that, MUT can even be used for fingerprint sensing [11]. Since the MUT consumes much less power than the optic sensor, it is probably the best candidate for the portable electronics, which has very strict limitation on power dissipation.

The conventional ultrasonic transducer is usually made of rigid bulk piezoelectric ceramic. Compared to the operation medium such as soft tissue, water or air, the rigid ceramic has much higher acoustic impedance. As the consequence of the huge impedance mismatch between the rigid ceramic and operation medium, over $90 \%$ of ultrasonic energy reflects back at the interface causing large losses [12]. Although a impedance matching layer can improve the transmitting efficiency, the $\lambda / 4$ thickness usually is very difficult to achieve, and sometimes is not even practical [13]. Unlike the conventional ultrasonic transducer, the MUT employs a flexural membrane for generating and receiving ultrasound waves. Since the flexural membrane is much softer than rigid ceramic, its acoustic impedance is closer to the operation medium. Thus, better impedance matching can be achieved, without the matching layer for MUTs, and so their transmitting efficiency is much higher than the conventional ultrasonic transducers [14].

Based on the transduction mechanism, the MUTs can be divided as two categories: the capacitive MUTs (cMUTs) and the piezoelectric MUTs (pMUTs). The cMUTs have been well developed since 1994 [15], and good impedance matching is shown by experimental results [14]. However, several inherent drawbacks limit their future applications. The cMUT requires an extremely small gap under the flexural membrane to achieve acceptable sensitivity. Fabrication of such small gap is complicated, expensive, and with very low yield. In addition, the operation of the cMUT requires very high voltage bias, which is usually over $100 \mathrm{~V}$. Such high voltage may bring safety issues for human body, especially for the implanted applications. The high voltage also limits its applications in portable devices. Moreover, the response of cMUT is highly non-linear, which requires sophisticated circuits for correction and signal processing.

As an alternative, pMUT overcomes all the inherent drawbacks of cMUT, and particularly it operates at a much lower voltage [16]. The pMUT seems to be a promising solution for 
future ultrasonic applications. Unfortunately, performances of previously reported pMUTs are much poorer than the expectation. Their coupling efficiency between electrical and mechanical domain is significantly lower than the prediction from analytical or finite elements models (FEM) [13], [16]-[19]. This problem is mainly attributed to the residual stress of the fabricated devices, because any residual stress may hamper the membrane vibration. Since fabrication of pMUT usually contains several high temperature processes, the residual stress in pMUT membrane can hardly be reduced. As the consequence, the sensitivity of the pMUT, in particular the transmitting sensitivity, is far from satisfactory [13], [20]. A few trials are made to address this stress issue. Mo et al. try to reduce the membrane buckling by removing the buried oxide layer. However, the improvement of device performance is quite limited [21]. Muralt et al. [16] try to optimize the fabrication process and use compressively stressed oxide layer to balance the lead zirconate titanate (PZT) layer with tensile stress. Although a relatively flat membrane is achieved through the stress compensation, the transmitting sensitivity is still unsatisfactory. Because the membrane vibration is very sensitive to the residual stress, even a non-obvious membrane deflection may drastically lower the transmitting sensitivity. A large DC bias is also introduced to balance the undesirable residual stress. Although the sensitivity is improved, this requires pMUT to be operated at high voltage similar as the cMUT [22], [23]. Therefore, researchers have to employ another bulk ceramic based ultrasonic transducer for ultrasound transmission, or even suggest the use of pMUT as only for ultrasound receiving for practical application [20]. Hence, it is highly critical to achieve very flat membrane, so as to achieve very high transmitting efficiency in order to be used as ultrasound transmitter.

PZT is widely adopted as the piezoelectric material for pMUT devices [24]-[32]. PZT usually has very high piezoelectric coefficient $d_{31}$ of $30-110 \mathrm{pC} / \mathrm{N}$ [33], [34]. However, deposition of PZT thin film requires a high temperature sintering at $600{ }^{\circ} \mathrm{C}$ for perovskite formation and hence is not CMOS compatible [30]. Therefore, aluminum nitride (AIN) as a CMOS compatible piezoelectric material has gained more interests in recent years [35]-[38], and AlN based pMUTs are also developed [2], [18], [39], [40]. The lower processing temperature $\left(<400{ }^{\circ} \mathrm{C}\right)$ allows AlN based pMUT to be monolithically fabricated with the ASICs. Although the $d_{31}$ of AlN is not as high as PZT, its lower dielectric constant enables a comparable receiving sensitivity with PZT based pMUTs [18]. In addition to its CMOS compatibility, the lower processing temperature of AlN also helps to reduce the residual thermal stress built up during the fabrication process. A high performance and low cost pMUT can thus be possibly achieved with AlN material.

In this paper, we design, fabricate and characterize an AlN based novel pMUT, aiming to overcome the residual stress and initial buckling issues. Leveraging on the nearly stressfree AlN thin film, dedicated top electrode configuration and integrated vacuum cavity, a perfectly zero-bending membrane is realized in our pMUT. The initial deflection of the membrane is measured as less than $0.005 \%$. Compared to the reference pMUT, where the membrane is slightly deflected, the transmitting sensitivity of the zero-bending pMUT is enhanced to above $450 \%$. The measured results match quite well with the simulation data, thereby indicating the pMUT almost achieves its ideal performance.

\section{Design Considerations}

\section{A. Influence of Residual Stress on Transmitting Sensitivity}

To predict the performance of pMUT, several analytical models are proposed [41]-[43]. The pMUT is modeled as a uniform circular thin plate with clamped boundary conditions. If the pMUT works at transmitting mode (i.e. the effects from external acoustic pressure can be neglected), the plate deflection $w(r)$ at a radial position $r$ can be described in the general form as:

$$
\begin{aligned}
D \nabla^{2} \nabla^{2} w(r)+\rho_{s} \frac{\partial^{2} w(r)}{\partial t^{2}} & =\nabla^{2} M^{p} \\
D & =\int \frac{y(Z) Z^{2}}{1-v(Z)^{2}} d Z \\
\rho_{s} & =\int \rho(Z) d Z
\end{aligned}
$$

where $D$ is the modulus of flexural rigidity, $\rho_{s}$ is the area plate density, $Y$ is the Young's modulus, $v$ is the poison's ratio, and $M^{p}$ is the piezoelectric moment induced by input voltage [42]. $Y$ and $v$ vary with z-direction because of the different materials at each layer. However, this model does not take residual stress into consideration. Influence of residual stress on pMUT performance is still not clear. Sammoura et al. firstly modify such model and introduce the effect of residual stress as [44], [45]:

$$
\begin{aligned}
D \nabla^{2} \nabla^{2} w(r)-T^{s} \nabla^{2} w(r)+\rho_{s} \frac{\partial^{2} w(r)}{d t^{2}} & =\nabla^{2} M^{p}+\nabla^{2} M^{s} \\
T^{s} & =\int \sigma(Z) d Z
\end{aligned}
$$

where $T^{s}$ is the overall plate tension caused by the residual stresses, $\sigma$ is the residual stress at different layers and $M^{s}$ is the residual moment about the neutral plane. Calculated results of the model indicate that the residual moment imbalance may cause buckling, and even lesser imbalance could adversely affect the transmitting sensitivity. With an overall plate tension of only $1000 \mathrm{~N} / \mathrm{m}$ (tensile), the deflection per voltage at the center of membrane reduces by $8.4 \%$ [44]. It is worth noting that the results do reveal that compressive stress can help to enhance the sensitivity. Some researchers also report that compressively stressed pMUTs with large upwards buckling have significantly higher transmitting [46] and receiving [47] sensitivities. However, large compressive stress and large buckling make the membrane very fragile. Intentionally introducing compressive stress could lower the yield rate, which is not preferred for volume fabrication. Considering both the performance and reliability, minimizing the overall plate tension and enabling flat membrane may be an effective and reliable way to enhance the transmitting sensitivity of pMUT. 
(a)

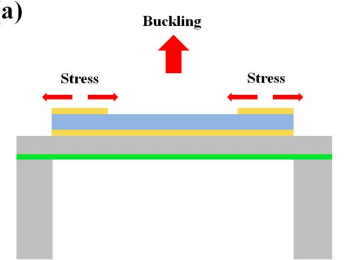

Conventional pMUT Design 1

Mo

(c)

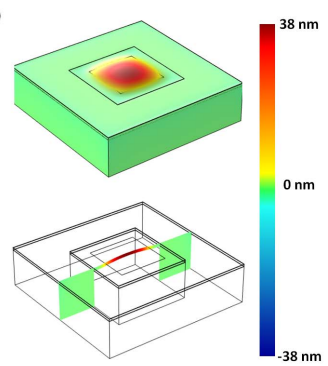

(b)

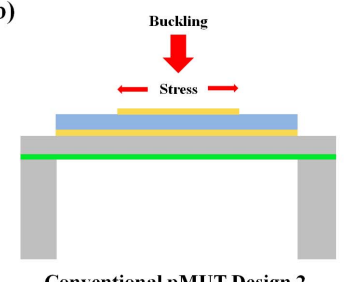

Si

(d)

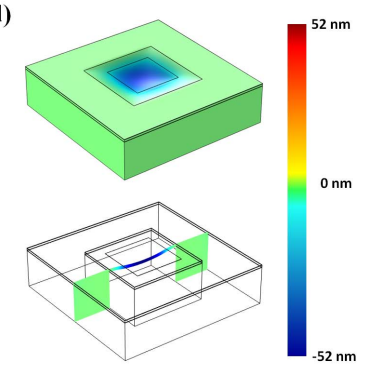

Fig. 1. Two typical conventional pMUT designs with: (a) Frame-like top electrode; (b) Central top electrode. The simulation results of residual stress induced buckling: (c) Design 1 and; (d) Design 2.

\section{B. Residual Stress of AlN Thin Film}

The residual stress of AlN thin film after deposition has been well studied recently [48]. Besides the deposition conditions, the residual stress of AlN thin film is largely dependent on its thickness. Generally the in-plane residual stress increases with the thickness, and the sign is changed from negative (compressive stress) to positive (tensile stress). For the AlN deposited on metal layer, the residual stress becomes zero at certain thickness of about $1 \mu \mathrm{m}$ [48]. Therefore, a nearly stress-free AlN thin film is possible by adjusting its thickness. In this work, a nearly stress-free AlN thin film is finally achieved at a thickness of $1 \mu \mathrm{m}$.

\section{Residual Stress of Conventional pMUT}

Only the stress-free AlN layer is not sufficient for a high performance pMUT, because the degradation of the transmitting sensitivity is attributed to the residual stress of every layer, according to Eq. (5). In addition, the stress distribution also changes because of the buckling, and the stress-free layers may become stressed after buckling. Finally, different designs and structures of pMUT affect the buckling as well. Analysis of the buckling and stress distribution should be considered case by case.

Figure 1 (a) and (b) show the two typical designs of pMUT, where the Design 1 has the frame-like electrode, while the Design 2 has the central electrode. According to previous studies, the curvature at the center of deflected membrane has the opposite sign from that at clamped borders. The sign changes at the position of about $65 \%$ of the radius [16]. Partially covered top electrode thus has the optimal performance, and both the frame-like electrode (outer) and the central electrode (inner) are identically the best [16], [49]. Therefore these two pMUT designs are adopted for the study.

TABLE I

Material Properties APPLied IN THE Finite Elements Model (FEM)

\begin{tabular}{ccccc}
\hline Material & $\begin{array}{c}\text { Young's } \\
\text { modulus }\end{array}$ & $\begin{array}{c}\text { Poisson's } \\
\text { ratio }\end{array}$ & $\begin{array}{c}\text { Residual } \\
\text { stress }\end{array}$ & $\begin{array}{c}\text { Thickness } \\
(\boldsymbol{\mu m})\end{array}$ \\
\hline $\mathrm{Mo}$ & $312 \mathrm{GPa}$ & 0.3 & $\begin{array}{c}470 \mathrm{MPa} \\
\text { (Tensile) }\end{array}$ & 0.2 \\
$\mathrm{AlN}$ & $340 \mathrm{GPa}$ & 0.24 & $\sim 0 \mathrm{MPa}$ & 1 \\
$\mathrm{Si}$ & $170 \mathrm{GPa}$ & 0.28 & $0 \mathrm{MPa}$ & 5 \\
$\mathrm{SiO}_{2}$ & $60 \mathrm{GPa}$ & 0.17 & $\begin{array}{c}-200 \mathrm{MPa} \\
\text { (Compressive) }\end{array}$ & 1 \\
\hline
\end{tabular}

A finite elements model (FEM) with a membrane size of $200 \mu \mathrm{m}$ is built using COMSOL Multiphysics software, where the solid mechanics physics is employed. The parameters used for simulation are summarized in Table I. Fig. 1 (c) and (d) show the simulated membrane deflections due to residual stresses for Design 1 and Design 2, respectively. With the stress-free AlN layer, the buckling is still not negligible. Such buckling may reversely affect the transmitting sensitivity. It is worth noting that the buckling directions of these two designs are opposite. Although the metal layer is very thin, it usually has very large tensile stress. So the buckling direction is mainly determined by the layout of top metal electrode. Simply removing the backside oxide layer [21], or adding additional oxide layer [16] can hardly alleviate the buckling, or may even worsen the situation. Therefore those trials to improve the pMUT performance are not very successful.

\section{Design of Zero-Bending pMUT}

The 3-D schematic drawings of the zero-bending pMUT are shown in the Fig. 2 (a) and (c), denoted as Device A. The frame-like top electrode is adopted for the zero-bending pMUT. Compared to the conventional design, an integrated vacuum cavity is fabricated under the membrane. As aforementioned, the pMUT with frame-like top electrode tends to bend upwards due to the residual stress. Meanwhile, the integrated vacuum cavity induces downwards atmospheric pressure, which pushes the membrane back. The initial bending of such pMUT therefore is compensated as shown in the Fig. 2 (d). Device B with the central top electrode (see Fig. 2 (b)) is taken as the reference. Since the pMUT with central top electrode tends to bend downwards and the vacuum induced atmospheric pressure is also the same direction, hence cannot balance the initial bending, but aggravates it, shown in Fig. 2 (e). Although the transmitting sensitivities of Device A and Device B should have been the same, the Device A with flat membrane is expected to have significantly higher sensitivity than the buckled Device B.

The membrane deflection of Device A is studied by simulation as well. As can be seen from Fig. 3 (a), the membrane of Device A becomes flat, and the deflection is minimized to less than $5 \mathrm{~nm}$. The stress distribution in the AlN layer of Device A along A- $\mathrm{A}^{\prime}$ direction is also extracted and shown in Fig. 3 (c). 
(a)

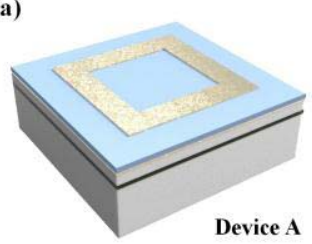

(b)

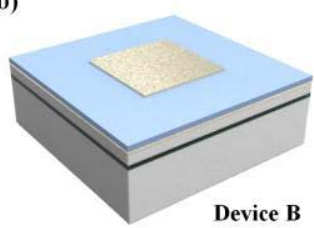

(d)

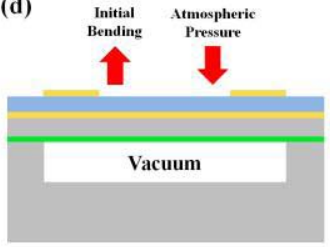

(c)
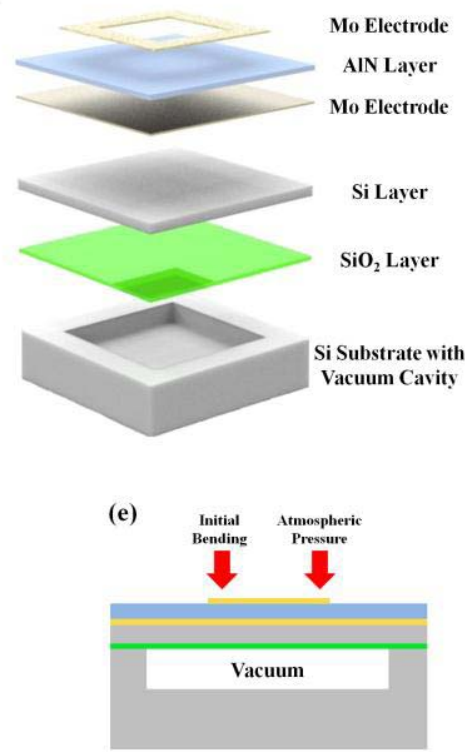

Si (a)

(b)

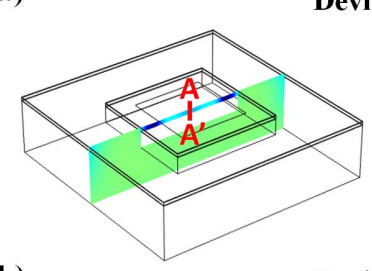

Device B

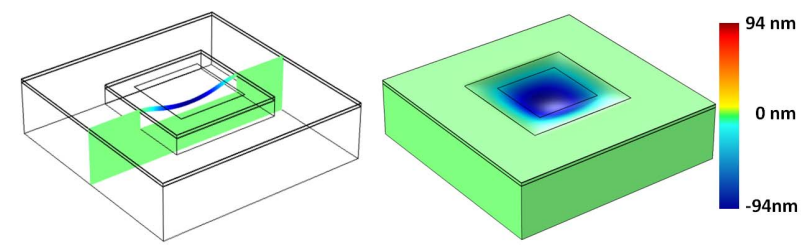

(c)

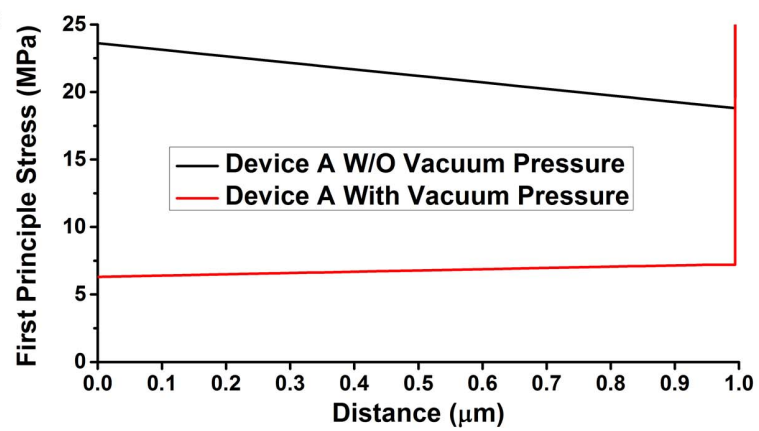

Fig. 2. 3-D schematic drawings of: (a) \& (c) the zero-bending pMUT, denoted as Device A; (b) Reference pMUT, denoted as Device B and; (d) \& (e) Comparison of Device A and Device B. Vacuum pressure can compensate the initial bending of Device A, but aggravate the initial bending of Device B.

The abrupt increasing of stress at $1 \mu \mathrm{m}$ is due to the extremely high initial stress in Mo layer. Despite that the residual stress of AlN layers is set to be zero (see Table I); the simulated stress in the AlN layer is still considerably high, because the initial buckling forces the AlN layer to be re-stressed. If the vacuum pressure is applied to the membrane and it becomes flat after the compensation, the stress in AlN layer significantly reduces to only $6 \mathrm{MPa}$. With the help of nearly stressfree AIN thin film, frame-like top electrode layout and integrated vacuum cavity, a zero-bending pMUT with minimized stress is possibly available. Fig. 3 (b) shows the reference pMUT (Device B) with applied vacuum pressure. As expected, it buckles with maximum central deflection of $94 \mathrm{~nm}$.

\section{DEVICE FABRICATION}

The fabrication of the zero-bending pMUT starts from a commercially purchasable cavity Silicon-on-insulator (SOI) wafer. Square-shaped cavities are firstly defined in the handle wafer using photolithography. The depth of the cavities is $5 \mu \mathrm{m}$. Then the cavity SOI wafer is formed by bonding the handle wafer with a $5 \mu \mathrm{m}$ device $\mathrm{Si}$ wafer in vacuum, shown in Fig. 4 (a). Prior to the deposition of Mo/AlN/Mo stack, a $20 \mathrm{~nm}$ AlN seeding layer is deposited by atomic layer deposition (ALD). This seeding layer helps to reduce the surface roughness of AlN layer, and consequently helps to improve the film quality of top Mo layer. Next the physical vapour deposition (PVD) is used to deposit the $0.2 \mu \mathrm{m} \mathrm{Mo} / 1 \mu \mathrm{m}$ AlN/0.2 $\mu \mathrm{m}$ Mo stack on the AlN seeding layer, shown in Fig. 4 (b). The top Mo layer is patterned using a $0.2 \mu \mathrm{m}$ plasma enhanced chemical vapour deposition (PECVD)

Fig. 3. Simulation results of membrane deflection: (a) Device A; (b) Device B and; (c) Stress distribution in the AIN layer of Device A along A-A' direction before and after vacuum pressure compensation.

silicon dioxide $\left(\mathrm{SiO}_{2}\right)$ layer as the hard mask. This hard mask is removed after Mo patterning using $\mathrm{HF}$ wet etching (Fig. 4 (c)). A layer of $0.7 \mu \mathrm{m} \mathrm{PECVD} \mathrm{SiO}_{2}$ layer is then deposited for isolation. This $\mathrm{SiO}_{2}$ is firstly etched by reactive ion etching (RIE), followed by AlN anisotropic dry etching to open the bottom-to-top contact via. Then the $\mathrm{SiO}_{2}$ layer is etched to open the top-to-top via, shown in Fig. 4 (d). Subsequently, a $0.7 \mu \mathrm{m} \mathrm{Al}$ is deposited and patterned to form the electrical connections and bonding pads (Fig. 4 (e)). Finally the oxide is blank etched by RIE dry etching to thin the membrane, shown in Fig. 4 (f). As the selectivity of $\mathrm{SiO}_{2}$ and $\mathrm{Al}$ is very high, no obvious attack to Al layer is observed after the process. Fig. 5 illustrates the fabricated zero-bending pMUT (Device A), as well as the reference pMUT (Device B). The integrated vacuum cavity is clearly shown in Fig. 5 (c), under the pMUT membrane.

\section{Device Characterization AND Discussion}

The fabricated pMUTs are firstly characterized using DHM-R2100 holographic MEMS analyzer by Lyncée Tec Ltd. This MEMS analyzer is able to capture both stationary and dynamic 3-D surface images of the device, with vertical resolution of sub nm level. The surface profile, deflection and dynamic motion of the MEMS device then can be extracted from such 3-D images. Fig. 6 (a) shows the 3-D images of Device A and Device B. As is expected, the Device A has a flat membrane, while the membrane of Device $B$ is 


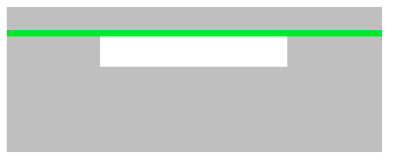

(b)

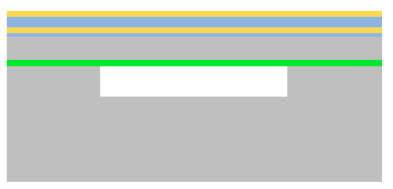

(c)

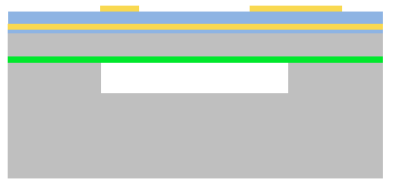

(f)
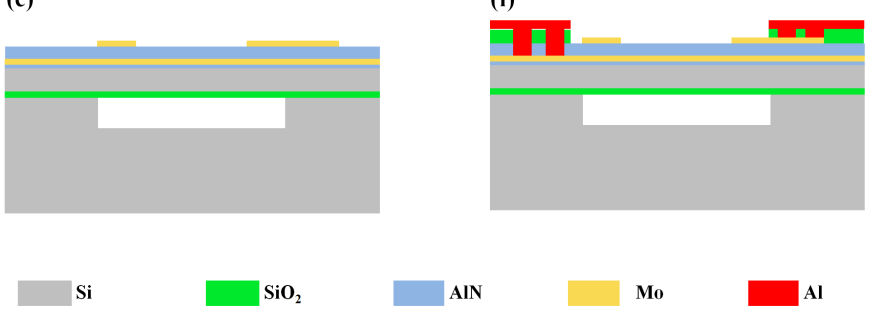

Fig. 4. Fabrication process flow of the zero-bending pMUT: (a) Formation of the cavity SOI wafer; (b) Deposition of Mo/AlN/Mo stack; (c) Patterning of top Mo layer; (d) Deposition of PECVD $\mathrm{SiO}_{2}$ for isolation, and $\mathrm{SiO}_{2}$ and $\mathrm{AlN}$ are etched to open contact via (bottom). The contact via (top) is then opened by etching $\mathrm{SiO}_{2}$; (e) Deposition and patterning of $\mathrm{Al}$ metal layer for electrical connections and to form bonding pads and; (f) Blank etching of oxide by RIE.

bent downwards. The $200 \mathrm{~nm}$ steps are the top electrodes. The surface profiles of both devices are also extracted and plotted in Fig. 6 (b). It is clearly shown that the membrane of Device A is perfectly flat and the $200 \mathrm{~nm}$ steps are the top electrode. The maximum deflection at the center of membrane is less than $10 \mathrm{~nm}$, or less than $0.005 \%$ (deflection/membrane size). Benefited from the stress-free AlN thin film, framelike top electrode and integrated vacuum cavity, the residual stress induced initial bending is successfully compensated. This extremely small initial bending therefore can be treated as zero bending. On the contrary, the membrane of Device B has a maximum deflection of $96 \mathrm{~nm}$. These measured surface profiles match with the simulation results in Fig. 3 very well.

The zero-bending pMUT (Device A) then is excited by $1 \mathrm{~V}$ electrical AC signals to evaluate its transmitting performance using the stroboscopic module. The displacement amplitude with frequency is plotted in Fig. 7 (a). Since all the displacement amplitudes are measured with $1 \mathrm{~V}$ excitation, the displacement amplitude per volt is also taken as transmitting sensitivity. The results of FEM simulation are shown as well for comparison. The piezoelectric devices physics is employed for this FEM, where the $1 \mu \mathrm{m}$ AlN layer is defined as the piezoelectric material with $d_{31}$ of $-1.72 \mathrm{pC} / \mathrm{N}$. The rest parts of the model are defined as linear elastic material with properties shown in Table I. To achieve reasonable results, loss and damping effects are also considered. The loss factor for electrical permittivity (dielectric loss) is set to be 0.01 , while the loss factor for damping (linear elastic material) is set to be 0.002 . The damping effect is mainly attributed to (a)

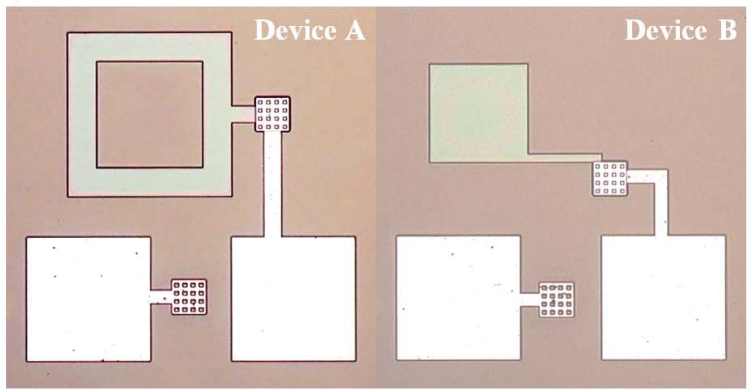

(b)

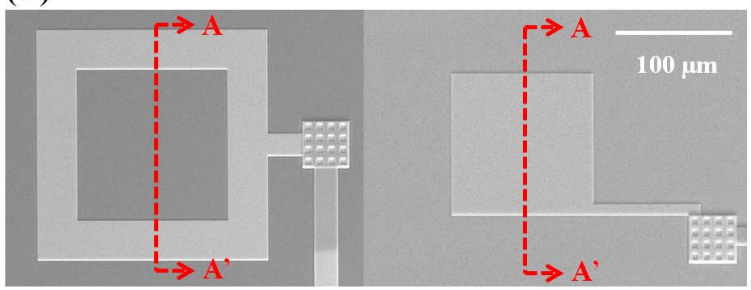

(c)

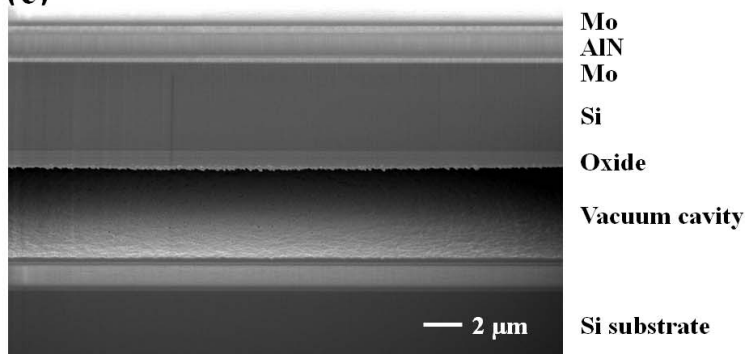

Fig. 5. The fabricated pMUT devices: (a) optical microscope (OM) images; (b) secondary electron microscope (SEM) images and; (c) cross-sectional view of SEM image along A-A' direction.

the anchor loss and air damping. It is worth noting that no initial stresses are considered in this FEM, and hence the simulated transmitting sensitivity is taken as the ideal value. The measured amplitude at the resonant frequency is $123 \mathrm{~nm}$, while the simulated amplitude is $129 \mathrm{~nm}$. It is clearly shown in Fig. 7 (a) that the measured curve is very close to the simulation result, which means a nearly ideal transmitting sensitivity is achieved for the zero-bending pMUT. Such pMUT achieves $94.5 \%$ of the ideal transmitting sensitivity.

In terms of the reference pMUT (Device B), however, the transmitting sensitivity is much poorer than the zero-bending pMUT, shown in Fig. 7 (b). As aforementioned, theoretically these two devices should have the same transmitting sensitivity, and is also proven seen through simulation results. Unfortunately, the reference pMUT fails to achieve a comparable transmitting sensitivity with the zero-bending pMUT, which largely deviates from the ideal performance. The displacement amplitude of the reference pMUT is measured as only $27 \mathrm{~nm}$, or $22 \%$ of the zero-bending pMUT. Such performance degradation is mainly because of the non-zero initial bending (Fig. 6). The slightly higher resonant frequency of the reference pMUT also indicates its membrane stress is larger than the zero-bending pMUT, as the membrane stiffness is increased due to the stress. Therefore, the zero-bending pMUT is proven to perform much better than 
(a)

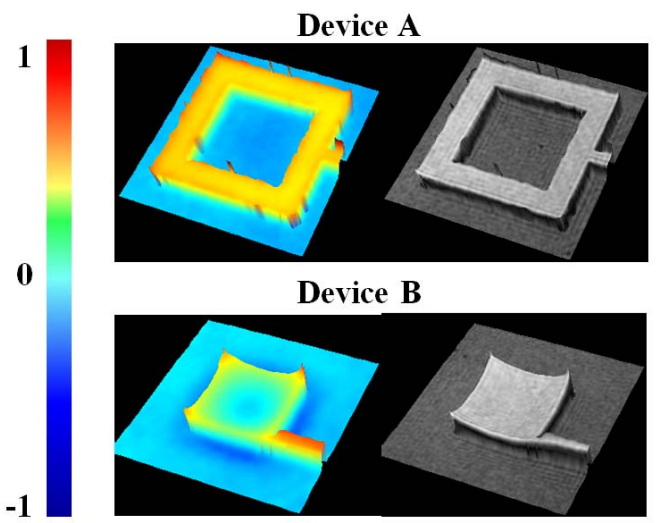

(b)
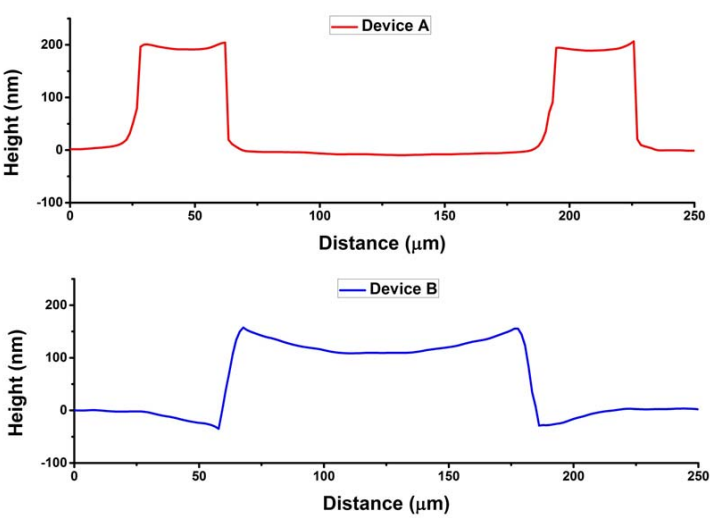

Fig. 6. (a) The 3-D images captured by holographic MEMS analyzer and; (b) The extracted surface profiles. Maximum deflection of the Device A is less than $10 \mathrm{~nm}$, which is considered as zero-bending, and the deflection of Device B is $96 \mathrm{~nm}$. The measured profiles match the simulation results very well.

TABLE II

COMPARISON OF TRANSMITTING SENSITIVITY

\begin{tabular}{cccc}
\hline Device & $\begin{array}{c}\text { Size } \\
(\boldsymbol{\mu m})\end{array}$ & $\begin{array}{c}\text { Transmitting } \\
\text { Sensitivity }(\mathbf{n m} / \mathbf{V})\end{array}$ & $\begin{array}{c}\text { Sensitivity per } \\
\text { Area }\left(\mathbf{n m} / \mathbf{V} / \mathbf{m m}^{2}\right)\end{array}$ \\
\hline This work & 200 & 123 & 3075 \\
Ref. [50] & 190 & 45 & 1587 \\
Ref. [18] & 350 & 210 & 2182 \\
Ref. [51] & 400 & 40 & 318 \\
Ref. [52] & 400 & 60 & 477 \\
Ref. [5] & 400 & 250 & 1989 \\
Ref. [40] & 1456 & 450 & 270 \\
Ref. [9] & 25 & 2.5 & $5092 *$ \\
\hline & \multicolumn{3}{c}{$*$ Extremely thin membrane }
\end{tabular}

the reference pMUT, with enhanced transmitting sensitivity of more than $450 \%$ with respect to the reference pMUT.

Table II summarizes previously reported AlN based pMUT devices. In general, the zero-bending pMUT presented in this work performs much better than most of other pMUTs. (a)

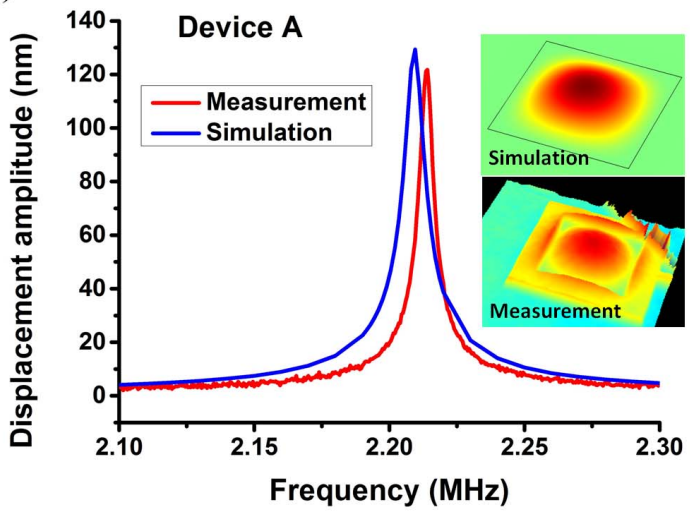

(b)

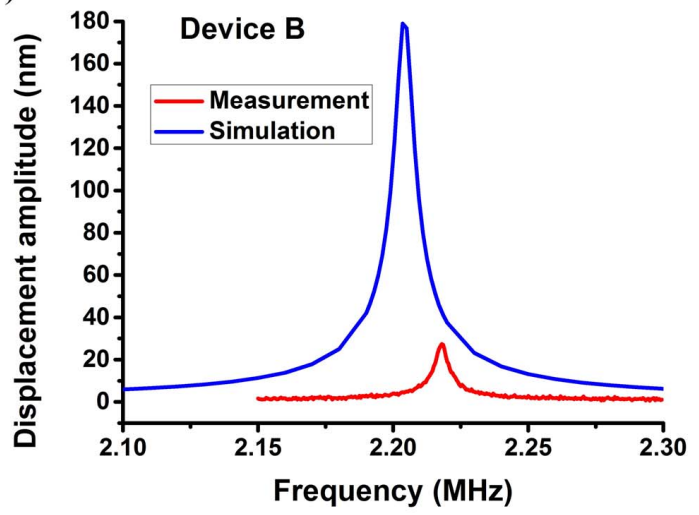

Fig. 7. Simulated and measured frequency responses of displacement amplitude with 1 V AC excitation for: (a) Device A and; (b) Device B. The inset shows the vibration mode shapes from both simulation and measurement.

Especially for the pMUTs reported in [50] and [51], those highly responsive pMUTs employ sophisticated 3-D membrane structure to further enhance the sensitivity, the sensitivity of the zero-bending pMUT is still twice and 10 times of the pMUTs in [50] and [51], respectively. It is worth noting that the pMUT in [9] has very high sensitivity. This may be because of the extremely thin membrane. Since its AlN and supporting $\mathrm{SiO}_{2}$ layers are only $0.75 \mu \mathrm{m}$ and $0.8 \mu \mathrm{m}$, its ideal transmitting sensitivity should be much higher than the zero-bending pMUT ( $1 \mu \mathrm{m}$ AlN, $5 \mu \mathrm{m} \mathrm{Si}$ and $1 \mu \mathrm{m} \mathrm{SiO})_{2}$ ). A FEM simulation is performed to investigate its ideal sensitivity using the above parameters. Simulation results indicate that the pMUT in [9] can potentially achieve a sensitivity of $14 \mathrm{~nm} / \mathrm{V}$, or $28520 \mathrm{~nm} / \mathrm{V} / \mathrm{mm}^{2}$. In fact, such pMUT only achieves $18 \%$ of its ideal sensitivity, which is significantly lower than the $94.5 \%$ of zero-bending pMUT.

Fig. 8 shows the impedance measurement results of the zero-bending pMUT, performed using Agilent 4294A precision impedance analyzer. The electromechanical coupling coefficient $k_{\text {eff }}^{2}$ can be derived from resonant frequency $f_{r}$ and anti-resonant frequency $f_{a}$ through following relation [26]:

$$
k_{e f f}^{2}=\frac{f_{a}^{2}-f_{r}^{2}}{f_{a}^{2}}
$$

This parameter directly reflects the electrical-mechanical energy conversion efficiency of the device. The in-air $k_{\text {eff }}^{2}$ is 
(a)

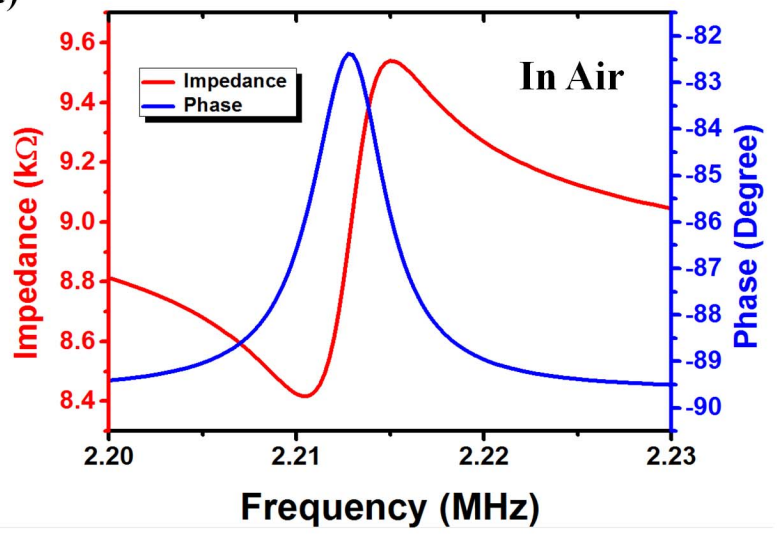

(b)

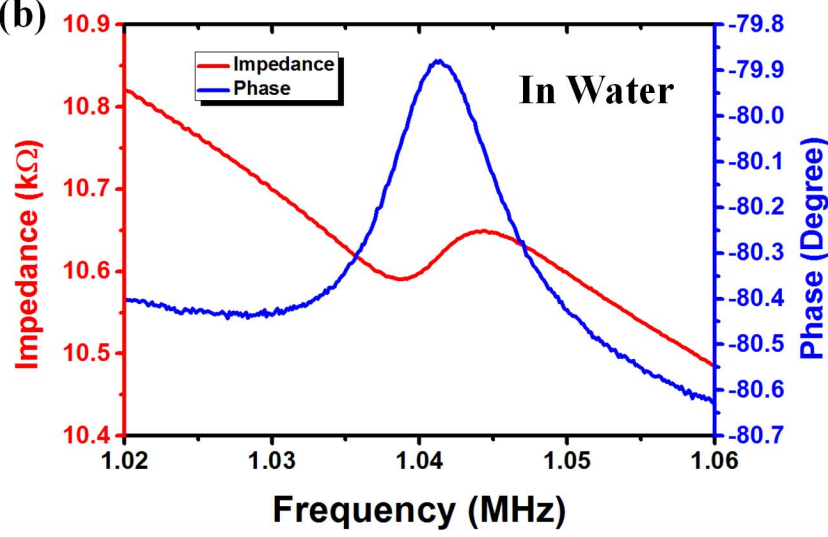

Fig. 8. The impedance measurement results of the zero-bending pMUT (Device A): (a) measured in air and; (b) measured in DI water.

calculated as $0.406 \%$. Such value is significantly better than a previously reported AlN based pMUT, where the measured coefficient is only $0.056 \%$ [18]. Considering the zero-bending pMUT is wire-bonded for testing, the coupling coefficient may be further improved by eliminating the parasitic capacitances from bonding pads and wires. As is shown in Fig. 8 (b), the zero-bending pMUT also performs well in DI-water. The resonant frequency drops to $1.04 \mathrm{MHz}$, which is still within the commonly used frequency range.

The pMUTs (frame-like top electrode) with different sizes are fabricated and studied as well. As can be seen from Fig. 9 (a), the resonant frequency decreases with the size as expected, and agrees very well with the simulation results. However, for the displacement amplitude, the measurement results start deviating from the simulation results at $300 \mu \mathrm{m}$, and such deviation increases with the membrane size (see Fig. 9 (b)). The $300 \mu \mathrm{m}$ and $400 \mu \mathrm{m}$ pMUTs are not able to achieve the best transmitting sensitivity as the $200 \mu \mathrm{m}$ pMUT. To investigate the reason of the performance degradation, the $300 \mu \mathrm{m}$ pMUT is taken as an example. Its surface profile is firstly extracted and shown in Fig. 9 (d). As is shown, such device is no longer a zero-bending pMUT, but with a maximum membrane deflection of $88 \mathrm{~nm}$. Because the vacuum force is proportional to the membrane area, it increases with the membrane size. Meanwhile, the residual stress in membrane remains a constant. Therefore the significant initial buckling is observed for $300 \mu \mathrm{m}$ pMUT. (a)

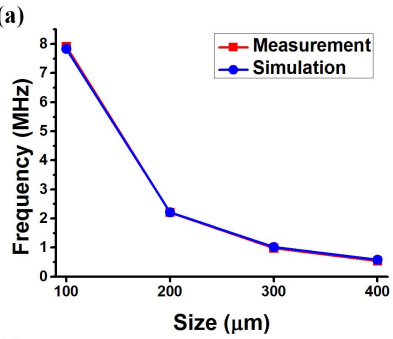

(c)
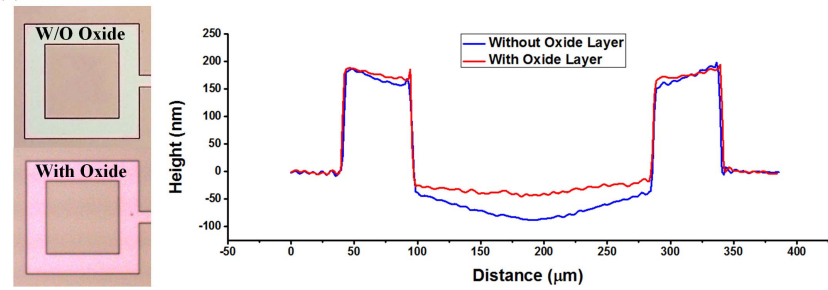

Fig. 9. Simulation and measurement results of the pMUTs (frame-like top electrode) with different sizes: (a) The resonant frequency and; (b) The displacement amplitude. The $300 \mu \mathrm{m}$ pMUTs with and without $0.7 \mu \mathrm{m}$ oxide layer: (c) Optical microscope images and; (d) measured surface profiles.

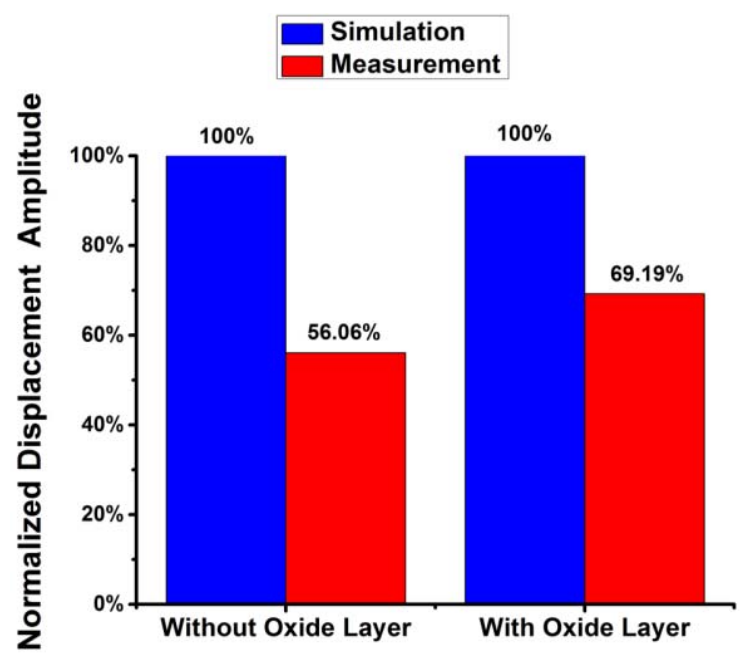

Fig. 10. Transmitting sensitivity comparison of $300 \mu \mathrm{m}$ pMUTs: without top oxide layer and with top oxide layer. The displacement amplitude is normalized to the simulation result, i.e. the ideal displacement amplitude.

The performance degradation is probably due to such initial buckling. As expected, the vacuum force of the $400 \mu \mathrm{m}$ pMUT is even larger, and hence the performance degradation becomes more severe for the $400 \mu \mathrm{m}$ pMUT.

To alleviate this performance degradation, another batch of devices is also fabricated. For these devices, the last step of process, i.e. blank etching of the top oxide layer, is skipped. Compared to previous pMUT, additional $0.7 \mu \mathrm{m}$ PECVD oxide layer is left on the surface, shown in Fig. 4 (e). As is shown in Fig. 9 (c), the color of the Mo electrode changes from green to purple, because of the oxide thin film. Since the oxide layer usually contains compressive stress, it forces the membrane to bend upwards. Therefore, the additional oxide layer may help to reduce the downwards initial bending. Fig. 9 (d) shows the surface profiles of $300 \mu \mathrm{m}$ pMUTs with and without oxide. With the help of the top oxide layer, the maximum membrane deflection reduces to $42 \mathrm{~nm}$. 
Although this pMUT is not perfectly zero-bending, the performance degradation should be alleviated due to the relatively flat membrane. Fig. 10 shows the transmitting sensitivity of the $300 \mu \mathrm{m}$ pMUTs, with and without top oxide layer. Benefited from the relatively flat membrane, the pMUT with oxide layer achieves $69.19 \%$ of ideal sensitivity, which is $13 \%$ better than the pMUT without oxide. Hence, if a larger membrane is required for lower operation frequency, additional top oxide layer may be applied to minimize the performance degradation.

\section{CONCLUSIONS}

Residual stress and initial buckling could suppress the transmitting sensitivity of pMUT. Although pMUT with large compressive stress and large upwards buckling exhibit high sensitivity, it may not be preferred for volume fabrication due to the fragility and lower yield rate. Several trials to minimize the stress and buckling are made by different research groups during last decades; there is still a room for innovative design with significant improvement. This is the very reason that pMUT has not been commercialized today due to that performance of current approaches is not satisfied, and hence the applications are very limited as well. In this paper, a new pMUT device is proposed. Leveraging on the stress-free AlN thin film, frame-like top electrode layout and the integrated vacuum cavity, a pMUT with zero-bending membrane is finally achieved. The transmitting sensitivity is measured as $123 \mathrm{~nm} / \mathrm{V}$, which is $450 \%$ better than that of reference pMUT with non-zero initial bending. Such pMUT achieves $94.5 \%$ of its ideal transmitting sensitivity. By thinning the supporting layer, i.e. the device Si layer, the transmitting performance may be further enhanced. In conclusion, the highly sensitive zero-bending pMUT is proven to be an effective and reliable way to overcome the residual stress and initial buckling issue. Therefore this work indicates a promising approach for optimization of various layout configuration of pMUT in order to bring performance of pMUT to the level of commercial interests.

\section{REFERENCES}

[1] Y. Lu and D. A. Horsley, "Modeling, fabrication, and characterization of piezoelectric micromachined ultrasonic transducer arrays based on cavity SOI wafers," J. Microelectromech. Syst., vol. 24, no. 4, pp. 1142-1149, 2015.

[2] B. Chen, F. Chu, X. Liu, Y. Li, J. Rong, and H. Jiang, "AlN-based piezoelectric micromachined ultrasonic transducer for photoacoustic imaging," Appl. Phys. Lett., vol. 103, no. 3, p. 031118, 2013.

[3] R. Przybyla et al., "A micromechanical ultrasonic distance sensor with $>1$ meter range," in Proc. 16th Int. Solid-State Sens., Actuators, Microsyst. Conf. (TRANSDUCERS), 2011, pp. 2070-2073.

[4] R. Przybyla, I. Izyumin, M. Kline, B. Boser, and S. Shelton, "An ultrasonic rangefinder based on an AlN piezoelectric micromachined ultrasound transducer," in Proc. IEEE Sensors, Nov. 2010, pp. 2417-2421.

[5] R. J. Przybyla et al., "In-air rangefinding with an aln piezoelectric micromachined ultrasound transducer," IEEE Sensors J., vol. 11, no. 11, pp. 2690-2697, Nov. 2011

[6] J. A. Jensen et al., "Recent advances in blood flow vector velocity imaging," in Proc. IEEE Int. Ultrason. Symp. (IUS), Oct. 2011, pp. 262-271.

[7] H. Fang, K. Maslov, and L. V. Wang, "Photoacoustic Doppler effect from flowing small light-absorbing particles," Phys. Rev. Lett., vol. 99, p. 184501 , Oct. 2007
[8] B. Khuri-Yakub, O. Oralkan, and M. Kupnik, "Next-gen ultrasound," IEEE Spectr., vol. 46, no. 5, pp. 44-54, May 2009.

[9] Y. Lu, A. Heidari, and D. A. Horsley, "A high fill-factor annular array of high frequency piezoelectric micromachined ultrasonic transducers," J. Microelectromech. Syst., vol. 24, no. 4, pp. 904-913, 2015.

[10] O. Rozen, S. T. Block, S. E. Shelton, R. J. Przybyla, and D. A. Horsley, "Air-coupled aluminum nitride piezoelectric micromachined ultrasonic transducers at $0.3 \mathrm{MHz}$ to $0.9 \mathrm{MHz}$," in Proc. 28th IEEE Int. Conf. Micro Electro Mech. Syst. (MEMS), Jan. 2015, pp. 921-924.

[11] Y. Lu et al., "Ultrasonic fingerprint sensor using a piezoelectric micromachined ultrasonic transducer array integrated with complementary metal oxide semiconductor electronics," Appl. Phys. Lett., vol. 106, no. 26, p. 263503, 2015.

[12] T. L. Szabo, Diagnostic Ultrasound Imaging: Inside Out. New York, NY, USA: Academic, 2004

[13] F. Akasheh, T. Myers, J. D. Fraser, S. Bose, and A. Bandyopadhyay, "Development of piezoelectric micromachined ultrasonic transducers," Sens. Actuators A, Phys., vol. 111, nos. 2-3, pp. 275-287, 2004.

[14] B. T. Khuri-Yakub and Ö. Oralkan, "Capacitive micromachined ultrasonic transducers for medical imaging and therapy," J. Micromech. Microeng., vol. 21, no. 5, p. 054004, 2011.

[15] M. J. Anderson, J. A. Hill, C. M. Fortunko, N. S. Dogan, and R. D. Moore, "Broadband electrostatic transducers: Modeling and experiments," J. Acoust. Soc. Amer, vol. 97, no. 1, pp. 262-272, 1995.

[16] P. Muralt et al., "Piezoelectric micromachined ultrasonic transducers based on PZT thin films," IEEE Trans. Ultrason., Ferroelect., Freq Control, vol. 52, no. 12, pp. 2276-2288, Dec. 2005.

[17] J. Cho, M. Anderson, R. Richards, D. Bahr, and C. Richards, "Optimization of electromechanical coupling for a thin-film PZT membrane: II. Experiment," J. Micromech. Microeng., vol. 15, no. 10, p. 1804, 2005.

[18] S. Shelton et al., "CMOS-compatible AlN piezoelectric micromachined ultrasonic transducers," in Proc. IEEE Int. Ultrason. Symp. (IUS), Sep. 2009, pp. 402-405.

[19] Y.-F. Wang et al., "Ultrasonic transducer array design for medical imaging based on MEMS technologies," in Proc. 3rd Int. Conf. Biomed. Eng. Informat. (BMEI), Oct. 2010, pp. 666-669.

[20] J. J. Bernstein et al., "Micromachined high frequency ferroelectric sonar transducers," IEEE Trans. Ultrason., Ferroelect., Freq. Control, vol. 44, no. 5, pp. 960-969, Sep. 1997.

[21] J.-H. Mo, A. L. Robinson, D. W. Fitting, F. L. Terry, and P. L. Carson, "Micromachining for improvement of integrated ultrasonic transducer sensitivity," IEEE Trans. Electron Devices, vol. 37, no. 1, pp. 134-140, Jan. 1990.

[22] P. Muralt and J. Baborowski, "Micromachined ultrasonic transducers and acoustic sensors based on piezoelectric thin films," J. Electroceram., vol. 12, nos. 1-2, pp. 101-108, 2004.

[23] B. Belgacem, F. Calame, and P. Muralt, "Piezoelectric micromachined ultrasonic transducers with thick PZT sol gel films," J. Electroceram., vol. 19, no. 4, pp. 369-373, 2007.

[24] Z. Wang, W. Zhu, O. K. Tan, C. Chao, H. Zhu, and J. Miao, "Ultrasound radiating performances of piezoelectric micromachined ultrasonic transmitter," Appl. Phys. Lett., vol. 86, no. 3, pp. 033508-1-033508-3, 2005.

[25] T. Matsushima, S. Xiong, H. Kawada, H. Yamanaka, and P. Muralt, "A highly sensitive $\mathrm{Pb}(\mathrm{Zr}, \mathrm{Ti}) \mathrm{O}_{3}$ thin film ultrasonic micro-sensor with a grooved diaphragm," IEEE Trans. Ultrason., Ferroelect., Freq. Control, vol. 54, no. 12, pp. 2439-2445, Dec. 2007.

[26] J. Jung, S. Kim, W. Lee, and H. Choi, "Fabrication of a twodimensional piezoelectric micromachined ultrasonic transducer array using a top-crossover-to-bottom structure and metal bridge connections," J. Micromech. Microeng., vol. 23, no. 12, p. 125037, 2013.

[27] Z. Wang, J. Miao, and W. Zhu, "Piezoelectric thick films and their application in MEMS," J. Eur. Ceram. Soc., vol. 27, nos. 13-15, pp. 3759-3764, 2007.

[28] Z. Wang, J. Miao, C. W. Tan, and T. Xu, "Fabrication of piezoelectric MEMS devices-from thin film to bulk PZT wafer," J. Electroceram., vol. 24, no. 1, pp. 25-32, 2010.

[29] Z. Wang et al., "Fabrication and characterization of piezoelectric micromachined ultrasonic transducers with thick composite PZT films," IEEE Trans. Ultrason., Ferroelect., Freq. Control, vol. 52, no. 12, pp. 2289-2297, Dec. 2005.

[30] Z. Wang, J. Miao, and W. Zhu, "Micromachined ultrasonic transducers and arrays based on piezoelectric thick film," Appl. Phys. A, vol. 91, no. 1, pp. 107-117, 2008.

[31] T. Wang, T. Kobayashi, and C. Lee, "Micromachined piezoelectric ultrasonic transducer with ultra-wide frequency bandwidth," Appl. Phys. Lett., vol. 106, no. 1, p. 013501, 2015. 
[32] A. Hajati et al., "Three-dimensional micro electromechanical system piezoelectric ultrasound transducer," Appl. Phys. Lett., vol. 101, no. 25, p. 253101, 2012.

[33] C. Lee, T. Itoh, R. Maeda, and T. Suga, "Characterization of micromachined piezoelectric PZT force sensors for dynamic scanning force microscopy," Rev. Sci. Instrum., vol. 68, no. 5, pp. 2091-2100, 1997.

[34] C. Lee, S. Kawano, T. Itoh, and T. Suga, "Characteristics of sol-gel derived PZT thin films with lead oxide cover layers and lead titanate interlayers," J. Mater. Sci., vol. 31, no. 17, pp. 4559-4568, 1996.

[35] T. Wang, X. Mu, P. Kropelnicki, A. B. Randles, and C. Lee, "Viscosity and density decoupling method using a higher order Lamb wave sensor," J. Micromech. Microeng., vol. 24, no. 7, p. 075002, 2014.

[36] J. H. Kuypers, C.-M. Lin, G. Vigevani, and A. P. Pisano, "Intrinsic temperature compensation of aluminum nitride Lamb wave resonators for multiple-frequency references," in Proc. IEEE Int. Freq. Control Symp., May 2008, pp. 240-249.

[37] C.-M. Lin, Y.-Y. Chen, V. V. Felmetsger, D. G. Senesky, and A. P. Pisano, "AlN/3C-SiC composite plate enabling high-frequency and high-Q micromechanical resonators," Adv. Mater, vol. 24, no. 20, pp. 2722-2727, 2012.

[38] C.-M. Lin, Y.-Y. Chen, V. V. Felmetsger, G. Vigevani, D. G. Senesky, and A. P. Pisano, "Micromachined aluminum nitride acoustic resonators with an epitaxial silicon carbide layer utilizing high-order Lamb wave modes," in Proc. IEEE 25th Int. Conf. Micro Electro Mech. Syst., (MEMS), Paris, France, Jan./Feb. 2012, pp. 733-736.

[39] H. Campanella, C. J. Camargo, J. López García, A. Daza, R. Urquiza, and J. Esteve, "Thin-film piezoelectric MEMS transducer suitable for middle-ear audio prostheses," J. Microelectromech. Syst., vol. 21, no. 6, pp. 1452-1463, 2012.

[40] B. A. Griffin, M. D. Williams, C. S. Coffman, and M. Sheplak, "Aluminum nitride ultrasonic air-coupled actuator," J. Microelectromech. Syst., vol. 20, no. 2, pp. 476-486, 2011.

[41] H. S. Choi, M. J. Anderson, J. L. Ding, and A. Bandyopadhyay, "A two-dimensional electromechanical composite plate model for piezoelectric micromachined ultrasonic transducers (pMUTs)," J. Micromech. Microeng., vol. 20, no. 1, p. 015013, 2010.

[42] K. Smyth, S. Bathurst, F. Sammoura, and S.-G. Kim, "Analytic solution for $N$-electrode actuated piezoelectric disk with application to piezoelectric micromachined ultrasonic transducers," IEEE Trans. Ultrason. Ferroelect., Freq. Control, vol. 60, no. 8, pp. 1756-1767, Aug. 2013.

[43] F. Sammoura and S.-G. Kim, "Theoretical modeling and equivalent electric circuit of a bimorph piezoelectric micromachined ultrasonic transducer," IEEE Trans. Ultrason., Ferroelect. Freq. Control, vol. 59, no. 5, pp. 990-998, May 2012.

[44] F. Sammoura, K. Smyth, S. Bathurst, and S.-G. Kim, "An analytical analysis of the sensitivity of circular piezoelectric micromachined ultrasonic transducers to residual stress," in Proc. IEEE Int. Ultrason. Symp. (IUS), Oct. 2012, pp. 580-583.

[45] F. Sammoura, K. Smyth, S.-G. Kim, and L. Lin, "An accurate equivalent circuit for the clamped circular multiple-electrode PMUT with residual stress," in Proc. IEEE Int. Ultrason. Symp. (IUS), Jul. 2013, pp. $275-278$.

[46] D. J. Morris, R. F. Need, M. J. Anderson, and D. F. Bahr, "Enhanced actuation and acoustic transduction by pressurization of micromachined piezoelectric diaphragms," Sens. Actuators A, Phys., vol. 161, nos. 1-2, pp. 164-172, 2010

[47] K. Yamashita, H. Nishimoto, and M. Okuyama, "Diaphragm deflection control of piezoelectric ultrasonic microsensors for sensitivity improvement," Sens. Actuators A, Phys., vol. 139, nos. 1-2, pp. 118-123, 2007.

[48] P. Pobedinskas et al., "Thickness dependent residual stress in sputtered AlN thin films," Thin Solid Films, vol. 522, pp. 180-185, Nov. 2012.

[49] S. Akhbari, F. Sammoura, C. Yang, M. Mahmoud, N. Aqab, and L. Lin, "Bimorph pMUT with dual electrodes," in Proc. 28th IEEE Int. Conf. Micro Electro Mech. Syst. (MEMS), Jan. 2015, pp. 928-931.

[50] S. Akhbari, F. Sammoura, S. Shelton, C. Yang, D. Horsley, and L. Lin, "Highly responsive curved aluminum nitride pMUT," in Proc. IEEE 27th Int. Conf. Micro Electro Mech. Syst. (MEMS), Jan. 2014, pp. 124-127.
[51] S. Akhbari, F. Sammoura, C. Yang, A. Heidari, D. Horsley, and L. Lin, "Self-curved diaphragms by stress engineering for highly responsive pMUT," in Proc. 28th IEEE Int. Conf. Micro Electro Mech. Syst. (MEMS), Jan. 2015, pp. 837-840.

[52] A. Guedes, S. Shelton, R. Przybyla, I. Izyumin, B. Boser, and D. A. Horsley, "Aluminum nitride pMUT based on a flexurallysuspended membrane," in Proc. 16th Int. Solid-State Sens., Actuators, Microsyst. Conf. (TRANSDUCERS), Jun. 2011, pp. 2062-2065.

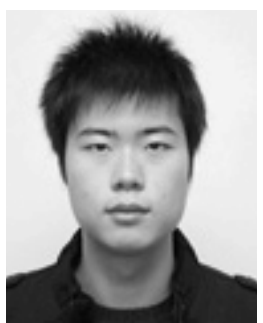

Tao Wang (M'14) received the B.Eng. degree from the School of Microelectronics and Solid-state Electronics, University of Electronic Science and Technology of China, Chengdu, China, in 2010, and the M.Sc. degree from the Department of Electrical and Computer Engineering, National University of Singapore, in 2012, where he is currently pursuing the Ph.D. degree with the ECE Department. His research interests are focused on piezoelectric MEMS devices.

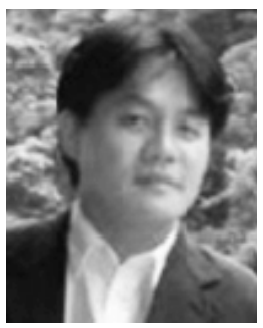

Chengkuo Lee (S'93-M'96) received the M.S degree in materials science and engineering from National Tsing Hua University, Hsinchu, Taiwan, in 1991, the M.S. degree in industrial and system engineering from Rutgers University, New Brunswick, NJ, in 1993, and the Ph.D. degree in precision engineering from The University of Tokyo, Tokyo, Japan, in 1996. He was a Foreign Researcher with the Nanometer Scale Manufacturing Science Laboratory, Research Center for Advanced Science and Technology, The University of Tokyo, from 1993 to 1996. He was with the Mechanical Engineering Laboratory, AIST, MITI, Japan, as a JST Research Fellow, in 1996. Thereafter, he became a Senior Research Staff Member with the Microsystems Laboratory, Industrial Technology Research Institute, Hsinchu. In 1997, he joined Metrodyne Microsystem Corporation, Hsinchu, and established the MEMS Device Division and the first micromachining fab for commercial purposes in Taiwan. He was the Manager of the MEMS Device Division from 1997 to 2000. He was an Adjunct Assistant Professor with the Electro-Physics Department, National Chiao Tung University, Hsinchu, in 1998, and the Institute of Precision Engineering, National Chung Hsing University, Taichung, Taiwan, from 2001 to 2005. In 2001, he co-founded Asia Pacific Microsystems, Inc., where he first became the Vice President of Research and Development, before becoming the Vice President of the Optical Communication Business Unit and a Special Assistant to the Chief Executive Officer in charge of international business and technical marketing for the microelectromechanical systems (MEMS) foundry service. From 2006 to 2009, he was a Senior Member of the Technical Staff with the Institute of Microelectronics, A*STAR, Singapore. $\mathrm{He}$ is currently an Associate Professor with the Department of Electrical and Computer Engineering, National University of Singapore, Singapore. $\mathrm{He}$ has co-authored the books Advanced MEMS Packaging (McGraw-Hill, 2010) and Micro and Nano Energy Harvesting Technologies (Artech House, 2014). $\mathrm{He}$ has contributed to more than 240 international conference papers and extended abstracts, and 170 peer-reviewed international journal articles in the fields of sensors, actuators, energy harvesting, MEMS, nanoelectromechanical systems, metamaterials, nanophotonics, and nanotechnology. He holds nine U.S. patents. 